\title{
Readmission following extracranial-intracranial bypass surgery in the United States: nationwide rates, causes, risk factors, and volume-driven outcomes
}

\author{
Kavelin Rumalla, BA, ${ }^{1}$ Visish M. Srinivasan, MD, ${ }^{2}$ Monica Gaddis, PhD, ${ }^{1}$ Peter Kan, MD, ${ }^{3}$ \\ Michael T. Lawton, MD, ${ }^{4}$ and Jan-Karl Burkhardt, MD \\ ${ }^{1}$ School of Medicine, University of Missouri-Kansas City, Missouri; ' 2 Department of Neurosurgery, Baylor College of Medicine, \\ Houston; ${ }^{3}$ Department of Neurosurgery, The University of Texas Medical Branch, Galveston, Texas; ${ }^{4}$ Department of \\ Neurosurgery, Barrow Neurological Institute, Phoenix, Arizona; and ${ }^{5}$ Department of Neurosurgery, Hospital of the University of \\ Pennsylvania, Philadelphia, Pennsylvania
}

OBJECTIVE Extracranial-intracranial (EC-IC) bypass surgery remains an important treatment option for patients with moyamoya disease (MMD), intracranial arteriosclerotic disease (ICAD) with symptomatic stenosis despite the best medical management, and complex aneurysms. The therapeutic benefit of cerebral bypass surgery depends on optimal patient selection and the minimization of periprocedural complications. The nationwide burden of readmissions and associated complications following EC-IC bypass surgery has not been previously described. Therefore, the authors sought to analyze a nationwide database to describe the national rates, causes, risk factors, complications, and morbidity associated with readmission following EC-IC bypass surgery for MMD, ICAD, and aneurysms.

METHODS The Nationwide Readmissions Database (NRD) was queried for the years 2010-2014 to identify patients who had undergone EC-IC bypass for MMD, medically failed symptomatic ICAD, or unruptured aneurysms. Predictor variables included demographics, preexisting comorbidities, indication for surgery, and hospital bypass case volume. A high-volume center (HVC) was defined as one that performed 10 or more cases/year. Outcome variables included perioperative stroke, discharge disposition, length of stay, total hospital costs, and readmission (30 days, 90 days). Multivariable analysis was used to identify predictors of readmission and to study the effect of treatment at HVCs on quality outcomes.

RESULTS In total, 2500 patients with a mean age of 41 years were treated with EC-IC bypass surgery for MMD (63.1\%), ICAD (24.5\%), or unruptured aneurysms (12.4\%). The 30- and 90 -day readmission rates were $7.5 \%$ and $14.0 \%$, respectively. Causes of readmission included new stroke (2.5\%), wound complications $(2.5 \%)$, graft failure $(1.5 \%)$, and other infection (1.3\%). In the multivariable analysis, risk factors for readmission included Medicaid/self-pay (OR 1.6, 95\% $\mathrm{Cl} 1.1-2.4$, vs private insurance), comorbidity score (OR 1.2, 95\% Cl 1.1-1.4, per additional comorbidity), and treatment at a non-HVC (OR 1.9, 95\% Cl 1.1-3.0). Treatment at an HVC (17\% of patients) was associated with significantly lower rates of nonroutine discharge dispositions ( $13.4 \%$ vs $26.7 \%, p=0.004)$, ischemic stroke within 90 days $(0.8 \%$ vs $2.9 \%$, $p=0.03$ ), 30 -day readmission ( $3.9 \%$ vs $8.2 \%, p=0.03)$, and 90 -day readmission $(8.6 \%$ vs $15.2 \%, p=0.01)$. These findings were confirmed in a multivariable analysis. The authors estimate that centralization to HVCs may result in 333 fewer nonroutine discharges ( $50 \%$ reduction), 12,000 fewer hospital days (44\% reduction), 165 fewer readmissions (43\%), and a cost savings of $\$ 15.3$ million ( $11 \%$ reduction).

CONCLUSIONS Readmission rates for patients after EC-IC bypass are comparable with those after other common cranial procedures and are primarily driven by preexisting comorbidities, socioeconomic status, and treatment at lowvolume centers. Periprocedural complications, including stroke, graft failure, and wound complications, occurred at the expected rates, consistent with those in prior clinical series. The centralization of care may significantly reduce perioperative complications, readmissions, and hospital resource utilization.

https://thejns.org/doi/abs/10.3171/2020.6.JNS202117

KEYWORDS cerebral bypass; readmission; nationwide; extracranial-intracranial; moyamoya disease; intracranial arteriosclerotic disease; ICAD; cerebral aneurysm; vascular disorders

ABBREVIATIONS EC-IC = extracranial-intracranial; HCUP = Healthcare Cost and Utilization Project; HVC = high-volume center; ICAD = intracranial arteriosclerotic disease; LOS = length of stay; MMD = moyamoya disease; NIS = National Inpatient Sample; NRD = Nationwide Readmissions Database; 30dRA = 1- to 30-day readmission; 90dRA = 1- to 90-day readmission.

SUBMITTED June 2, 2020. ACCEPTED June 19, 2020.

INCLUDE WHEN CITING Published online November 6, 2020; DOI: 10.3171/2020.6.JNS202117. 
$\mathrm{E}$ XTRACRANIAL-INTRACRANIAL (EC-IC) bypass surgery is performed to augment or preserve cerebral blood flow, depending on the treated disease. The most common indications for bypass surgery are moyamoya disease (MMD) or syndrome, intracranial arteriosclerotic disease (ICAD) with symptomatic high-grade vessel stenosis or occlusion despite the best medical therapy, or complex aneurysms not amenable to other open surgical or endovascular techniques. ${ }^{1}$ Besides the classic EC-IC bypass technique without an interposition graft (type 1), other EC-IC bypass techniques with interposition grafts (type 2) or IC-IC bypass techniques (type 3) exist and can be applied depending on the individual case. ${ }^{2} \mathrm{Be}-$ cause of the technical complexity of the procedure and the fragile patient population in need of a bypass, the results of previous clinical trials have been mixed, and overall complications and morbidity have been high. ${ }^{3,4}$ Therefore, it has been recommended that bypass surgery be performed at high-volume centers (HVCs) by experienced surgeons.

In addition to being cognizant of patient safety concerns, it is also important to minimize complications to avoid unplanned readmissions, which are costly to patients, hospitals, and insurance companies and can interfere with the benefits of surgical interventions. In recent years, federal regulations linking readmissions to hospital reimbursement penalties have increased the attention given to this quality metric. Prior studies have described the nationwide burden of readmissions following several neurosurgical procedures, including elective aneurysm clipping, ${ }^{5}$ craniotomy for brain tumor, ${ }^{6}$ acoustic neuroma, ${ }^{7}$ and epilepsy surgery. ${ }^{8}$ To date, however, the nationwide rates and reasons for readmission following cerebral bypass surgery have not been described. Presumably, readmissions following cerebral bypass surgery are likely due to sequelae of underlying medical comorbidity (e.g., stroke in ICAD), complications due to the surgical procedure (e.g., wound infection, graft failure, hemorrhage), or premature discharge of patients with a poor functional status. Therefore, we sought to analyze a nationwide database to describe the national rates, causes, risk factors, complications, and morbidity associated with readmission following EC-IC bypass surgery for MMD, ICAD, and aneurysms.

\section{Methods \\ Data Source}

The Nationwide Readmissions Database (NRD) is the largest all-payer inpatient database in the US and contains patient records from over 2000 hospitals representing approximately $56 \%$ of all US hospitalizations. Unlike its predecessors, such as the National Inpatient Sample (NIS), the NRD contains patient linkage numbers allowing for tracking of individual patients across multiple hospitalizations. Within the NRD, each diagnosis or procedure is identified using ICD-9-CM codes. The NRD is in the public domain, de-identified, and classified as nonhuman subject research, and thus this study was exempt from institutional review board approval. More details about the NRD are located at https://www.hcup-us.ahrq.gov/nrdoverview.jsp.

\section{Study Design}

The present study was a population-based longitudinal analysis of the NRD (years 2010-2014) of the Healthcare Cost and Utilization Project (HCUP), Agency for Healthcare Research and Quality. We refrained from querying the NRD for the years of the ICD-9 to ICD-10 transition period (2015-2016) to decrease inaccuracies due to a billing coder learning curve and variations in institutional practices. The study design conformed with the STROBE guideline checklist.

\section{Participants}

The index (surgery) cohort was identified by querying the NRD for January to September of each year (2010 through 2014) for patients who had undergone EC-IC bypass (ICD-9-CM procedure code 39.28) for MMD (437.5), ICAD $(433.10,433.11,433.30,434.90,434.91)$, or an unruptured cerebral aneurysm (437.3). This ICD-9-CM coding algorithm has been utilized in several prior neurosurgical publications. ${ }^{9-11}$

\section{Setting}

The readmission variable was computed per HCUP guidelines using NRD_visitLink (patient linkage number for linking hospital visits for the same patient across hospitals), NRD_DaysToEvent (timing variable), and lengthof-stay (LOS) variables. A patient's first rehospitalization following the bypass hospitalization was counted as a readmission. The NRD data set does not track patient visits across years. Initial surgical (index) encounters were only queried for patients discharged from January 1 to September 30 of each calendar year. This allowed for a complete 90-day postdischarge window for identifying readmissions. Hospitalizations with the admission type indicator of "elective" were excluded. Same-day revisits and transfers were collapsed into a single discharge record and were not counted as readmissions.

\section{Predictor Variables}

The data set was abstracted for age, sex, payer status, preexisting comorbidities, severity of illness, hospital type, indication for procedure, neurosurgical complications, medical complications, and discharge disposition (adverse $=$ disposition other than home). Hospitals were stratified by EC-IC bypass case volume. An HVC was defined as one that performed 10 or more cases per year. Neurosurgical complications included iatrogenic stroke, iatrogenic hematoma/hemorrhage, graft failure, and wound complication (e.g., seroma, site infection, dehiscence). Medical complications included respiratory, renal, and cardiovascular complication and non-CNS infection (e.g., pneumonia, septicemia, urinary tract infection).

\section{Outcome Variables}

The primary outcome variable was time to readmission, which was computed using the method described by HCUP. Time to readmission was recoded into the binary outcome variables "1- to 30-day readmission" (30dRA) and "1- to 90-day readmission" (90dRA). These quality metric designations have been readily utilized in the neu- 
TABLE 1. Causes of readmission within 90 days of EC-IC bypass surgery

\begin{tabular}{|c|c|c|}
\hline Variable & $30 \mathrm{dRA}$ & 90dRA \\
\hline Total no. of patients analyzed & 2500 & 2500 \\
\hline Total readmission rate, no. (\%) & $187(7.5)$ & $351(14.0)$ \\
\hline \multicolumn{3}{|l|}{$\begin{array}{l}\text { Most common diagnoses at } \\
\text { readmission, no. }(\%)\end{array}$} \\
\hline MMD & $72(2.9)$ & $189(7.6)$ \\
\hline Ischemic stroke & $32(1.3)$ & $63(2.5)$ \\
\hline $\begin{array}{l}\text { Wound complication (includes } \\
\text { infection) }\end{array}$ & $29(1.2)$ & $63(2.5)$ \\
\hline Mechanical complication of graft & $22(0.9)$ & $37(1.5)$ \\
\hline Hemorrhagic stroke & ${ }^{*}$ & $16(0.6)$ \\
\hline $\begin{array}{l}\text { Infection (septicemia, } \\
\text { pneumonia, UTI) }\end{array}$ & $15(0.6)$ & $32(1.3)$ \\
\hline Syncope/convulsions & $18(0.7)$ & $36(1.4)$ \\
\hline Sickle cell complications & $11(0.4)$ & $26(1.0)$ \\
\hline \multicolumn{3}{|l|}{$\begin{array}{l}\text { Most common procedures at } \\
\text { readmission, no. }(\%)\end{array}$} \\
\hline Angiogram & $31(1.2)$ & $56(2.2)$ \\
\hline Additional EC-IC bypass & $12(0.5)$ & $19(0.8)$ \\
\hline \multicolumn{3}{|l|}{$\begin{array}{l}\text { In-hospital outcome, readmission } \\
\text { visit }\end{array}$} \\
\hline Mean LOS in days (SD) & $5.2(8.7)$ & $10.7(11.3)$ \\
\hline Mean total costs in US\$ (SD) & $16,240(26,760)$ & $46,494(41,114)$ \\
\hline \multicolumn{3}{|l|}{ Discharge disposition, no. (\%) } \\
\hline Routine & $134(5.4)$ & $232(9.3)$ \\
\hline Long-term facility & $27(1.1)$ & $54(2.2)$ \\
\hline Home health care & $24(1.0)$ & $49(2.0)$ \\
\hline Mortality & ${ }^{*}$ & ${ }^{*}$ \\
\hline Other & * & * \\
\hline
\end{tabular}

UTI = urinary tract infection.

* Below HCUP minimum of 11 cases per cell.

rosurgical literature. ${ }^{5-8,12-17}$ Secondary outcomes of interest included discharge disposition, mean LOS (days), and mean total hospital costs (US\$).

\section{Statistical Analysis}

The complex samples functions in IBM SPSS Statistics software (version 26; IBM Corp.) were used to perform survey-adjusted analyses. The time-to-readmission variable was recoded into the binary outcome variables 30dRA and 90dRA. We generated nationwide estimates for the rates and causes of readmission in each group. To identify predictors of 90dRA, we constructed binary logistic regression models to generate odds ratios and 95\% confidence intervals. Clinically significant variables with at least 30 cases per cell and $p<0.05$ per chi-square analysis were included in the multivariable models. Statistical significance was set as an alpha of 0.05 . Clinical significance was determined by considering the width of confidence intervals and effect size.

We quantified the relationship between treatment at HVCs and outcomes in additional univariable and mul- tivariable models. LOS and total hospital cost were dichotomized into the binary outcome variables "prolonged LOS" (> 75th percentile for LOS, 10 days) and "increased total costs" ( $>75$ th percentile for costs, $\$ 52,851)$. Similarly, discharge disposition was dichotomized into "nonroutine" and "routine." The multivariable models were adjusted for patient age, payer type, preexisting comorbidities, and indication for surgery. We estimated the theoretical improvement in outcomes if all patients were treated at HVCs instead of non-HVCs.

\section{Results \\ Participants}

In total, 2500 patients were treated with EC-IC bypass for MMD (63.1\%, $\mathrm{n}=1576)$, ICAD $(24.5 \%, \mathrm{n}=613)$, or unruptured aneurysms $(12.4 \%, \mathrm{n}=310)$. The index visit was the surgical hospitalization. The mean $( \pm$ SD) patient age was $41 \pm 18.6$ years, $65 \%$ of the patients were female, and $58 \%$ of the patients had two or more comorbidities. As expected, the vast majority of procedures were performed at large metropolitan teaching hospitals (97\%).

A subgroup analysis revealed significant differences in patient characteristics by indication for bypass surgery. The MMD subgroup was significantly younger (mean age 34 years) than the ICAD (53 years) or aneurysm (54 years) subgroups $(\mathrm{p}<0.001)$. The average comorbidity score was increased in the ICAD (2.7) and aneurysm (2.5) groups compared to that in the MMD group (1.7; $p<0.001)$. Individual comorbidities were more common in the ICAD cohort and included tobacco smoking, dyslipidemia, diabetes, chronic hypertension, and peripheral vascular disease (all $\mathrm{p}<0.05$ ).

\section{Perioperative Complications and Index Discharge Outcomes}

The average LOS was 9.9 days $( \pm 14.4)$ and mean total cost of hospitalization was $\$ 46,779( \pm \$ 46,128)$. Most patients were discharged routinely to home $(76 \%)$ versus a long-term care facility (13.4\%), home health (10.3\%), or short-term postacute care $(0.7 \%)$. Overall mortality was $2 \%$, and medical complications occurred in $18.1 \%$ of patients including infection (9.6\%), respiratory distress/failure $(7.3 \%)$, and deep vein thrombosis/pulmonary embolus $(6.1 \%)$. The rate of iatrogenic stroke or hemorrhage was significantly higher in patients undergoing bypass surgery for an unruptured aneurysm $(25.7 \%)$ than in patients treated for MMD $(2.7 \%)$ or ICAD $(4.3 \%$; $\mathrm{p}<0.001)$. Other perioperative complications included graft failure (1.4\%) and wound complications (1.2\%). The rate of adverse discharge disposition (short-term nursing, long-term facility, or home health) was significantly higher in patients readmitted following bypass for ICAD (35.4\%) or aneurysm $(52.8 \%)$ than in those readmitted after bypass for MMD $(14.5 \% ; \mathrm{p}<0.001)$.

\section{Readmission Rates and Causes}

The 30dRA and 90dRA rates were $7.5 \%$ and $14.0 \%$, respectively, and varied by clinical indication for bypass at $7.6 \%$ and $12.3 \%$ for MMD, $8.6 \%$ and $19.2 \%$ for ICAD, and $4.6 \%$ and $12.7 \%$ for aneurysm, respectively (Tables 1 
TABLE 2. Perioperative complications and readmissions by surgical indication for EC-IC bypass surgery

\begin{tabular}{|c|c|c|c|c|c|}
\hline Variable & All & MMD & ICAD & Aneurysm & $p$ Value \\
\hline Total no. of patients (\%) & 2500 & $1577(63.1)$ & $613(24.5)$ & $310(12.4)$ & \\
\hline Mean age in yrs (SD) & $41.1(18.6)$ & $33.9(16.4)$ & $53.3(15.8)$ & $54.2(14.9)$ & $<0.001$ \\
\hline Comorbidity score (SD) & $1.9(1.6)$ & $1.7(1.4)$ & $2.7(1.9)$ & $2.5(1.9)$ & $<0.001$ \\
\hline \multicolumn{6}{|l|}{ Index visit } \\
\hline Medical complication (\%) & 18.1 & 11.5 & 20.6 & 46.5 & $<0.001$ \\
\hline Respiratory & 7.3 & 2.9 & 11.1 & 22.1 & $<0.001$ \\
\hline Infection & 9.6 & 5.5 & 9.8 & 30.0 & $<0.001$ \\
\hline DVT/PE & 6.1 & 3.9 & 5.7 & 18.0 & $<0.001$ \\
\hline Urinary & 1.1 & 0.8 & 1.8 & $*$ & 0.386 \\
\hline Cardiovascular & 0.9 & * & * & * & 0.032 \\
\hline latrogenic stroke (\%) & 5.9 & 2.7 & 4.3 & 25.7 & $<0.001$ \\
\hline Graft complication (\%) & 1.4 & * & 2.4 & 3.6 & 0.003 \\
\hline Wound complication (\%) & 1.2 & * & * & 6.3 & $<0.001$ \\
\hline Adverse discharge $(\%) \dagger$ & 24.4 & 14.5 & 35.4 & 52.8 & $<0.001$ \\
\hline Mean LOS in days (SD) & $9.9(13.4)$ & $7.4(12.6)$ & $13.0(15.7)$ & $17.9(16.9)$ & $<0.001$ \\
\hline Mean total costs in US\$ (SD) & $46,779(46,128)$ & $38,427(34,390)$ & $53,298(55,290)$ & $85,181(62,759)$ & $<0.001$ \\
\hline \multicolumn{6}{|l|}{ Readmission visit } \\
\hline $30 \mathrm{dRA}(\%)$ & 7.5 & 7.6 & 8.6 & 4.6 & 0.47 \\
\hline Wound complication & 1.2 & 1.0 & 1.8 & * & 0.62 \\
\hline Graft failure & 0.9 & * & * & * & * \\
\hline Ischemic stroke & 1.3 & 1.3 & 1.8 & * & 0.43 \\
\hline Hemorrhagic stroke & * & * & * & * & * \\
\hline Repeat EC-IC bypass & 0.5 & 0.5 & ${ }^{*}$ & * & * \\
\hline $90 \mathrm{dRA}(\%)$ & 14.0 & 12.3 & 19.2 & 12.7 & 0.023 \\
\hline Wound complication & 2.5 & 2.0 & 4.4 & * & 0.37 \\
\hline Graft failure & 1.5 & 0.7 & 1.6 & 0.6 & $<0.001$ \\
\hline Ischemic stroke & 2.5 & 2.0 & 5.2 & * & 0.09 \\
\hline Hemorrhagic stroke & 0.6 & * & * & * & * \\
\hline Repeat EC-IC bypass & 0.8 & 1.2 & * & * & * \\
\hline Adverse discharge (\%)† & 37.6 & 17.0 & 35.2 & 70.1 & $<0.001$ \\
\hline Mortality (\%) & 0.5 & * & * & * & * \\
\hline
\end{tabular}

DVT = deep vein thrombosis; $\mathrm{PE}=$ pulmonary embolus.

Boldface type indicates statistical significance.

* Below HCUP minimum of 11 cases per cell.

$\dagger$ Discharge other than home.

and 2). Readmission for ischemic stroke, wound complications, graft failure, non-CNS infection, and syncope occurred at approximately equal frequencies in the $30 \mathrm{dRA}$ and 90dRA cohorts. However, repeat bypass procedures primarily occurred within 30 days.

\section{Readmission and Postoperative Complications}

The overall rate of readmission for acute ischemic stroke was $2.5 \%$ with a higher rate in patients operated on for ICAD than in those treated for MMD $(5.2 \%$ vs $2.0 \%$, p $=0.09$ ). Graft failure occurred in $1.5 \%$ of patients overall and was more commonly found in patients operated on for MMD $(0.7 \%)$ and ICAD (1.6\%) than in those treated for aneurysms $(0.6 \% ; \mathrm{p}<0.001)$. Wound complications (e.g., seroma, dehiscence) occurred in $2.5 \%$ of patients overall. The rate of additional EC-IC bypass was $0.8 \%$ overall (n
= 19), and all cases were in the MMD cohort. However, we cannot exclude the possibility that these cases represent bypass of the contralateral side in MMD patients (Table 2).

Readmission visits were associated with additional adverse outcomes. The average cost of readmission within 30 and 90 days was $\$ 16,240 \pm \$ 26,760$ and $\$ 46,494 \pm \$ 41,114$, respectively (Table 1). The rate of adverse discharge disposition was $37.6 \%$ overall and $35.2 \%$ in the ICAD group and $70.1 \%$ in the aneurysm group, as compared to $17.0 \%$ in the MMD group. There were 12 readmission-associated deaths $(0.5 \%$; Table 2$)$.

\section{Risk Factors for Readmission}

In the multivariable analysis, risk factors associated with an increased likelihood of 90dRA included Medicaid/self-pay versus private insurance (OR 1.6, 95\% CI 
TABLE 3. Multivariable analysis of risk factors associated with 90dRA following EC-IC bypass surgery

\begin{tabular}{lccc}
\hline \multicolumn{1}{c}{ Predictor Variable } & OR & $95 \% \mathrm{Cl}$ & $\mathrm{p}$ Value \\
\hline Age, per yr increase & 1.01 & $0.99-1.02$ & 0.313 \\
\hline $\begin{array}{l}\text { Comorbidity score (per } \\
\text { additional comorbidity) }\end{array}$ & 1.21 & $1.09-1.35$ & $\mathbf{0 . 0 0 1}$ \\
\hline $\begin{array}{l}\text { Insurance payer } \\
\text { Private }\end{array}$ & & & \\
\hline Medicare & Reference & & \\
\hline Medicaid/self-pay & 1.13 & $0.65-1.95$ & \\
\hline Primary diagnosis & 1.63 & $1.13-2.36$ & \\
\hline MMD & & & 0.345 \\
\hline ICAD & Reference & & \\
\hline Aneurysm & 1.13 & $0.71-1.80$ & \\
\hline Hospital case vol & 0.68 & $0.36-1.29$ & \\
\hline Non-HVC (1-9 cases/yr) & Reference & & 0.016 \\
\hline HVC (10+ cases/yr) & 0.54 & $0.33-0.89$ & \\
\hline
\end{tabular}

Boldface type indicates statistical significance.

1.1-2.4), comorbidity score (OR 1.2, 95\% CI 1.1-1.4, per additional comorbidity), and treatment at a non-HVC (OR 1.85, 95\% CI 1.12-3.03; Table 3). Individual comorbidities were collapsed into a composite variable for multivariable analysis because of small cell numbers.

\section{Hospital EC-IC Bypass Case Volume and Quality Outcomes}

Hospitals were stratified into percentile groups by annual bypass case volume (Table 4). Increasing case volume was associated with progressively decreasing rates of ischemic stroke within 90-day and all-cause readmission rates (Table 5). We defined an HVC as an institution performing 10 or more EC-IC bypasses per year. Only $17.4 \%$ of patients were treated at HVCs. Patients treated at HVCs had significantly lower rates of nonroutine discharge disposition ( $13 \%$ vs $27 \%, p=0.004)$, average LOS (6.1 vs 10.9 days, $\mathrm{p}<0.001)$, and readmissions within 30 days $(3.9 \%$ vs $8.2 \%, \mathrm{p}=0.03)$ and 90 days $(8.6 \%$ vs $15.2 \%, \mathrm{p}=0.01$; Table 6).

Our multivariable analysis, adjusted for potential confounders, confirmed our univariable results with negligible changes to the effect sizes (Table 7). Treatment at HVCs was associated with a decreased likelihood of nonroutine discharge (OR 0.54, 95\% CI 0.34-0.86), prolonged
LOS (OR 0.57, 95\% CI 0.33-0.98), 30dRA (OR 0.43, 95\% CI $0.20-0.91$ ), and 90dRA (OR 0.54, 95\% CI 0.33-0.89). There was no significant difference in the average cost of hospitalization between HVCs and non-HVCs.

Given the strong volume-outcome relationship, further statistical models were developed to estimate the impact of the centralization of care (Table 8). The relationship between case volume and outcome was established in both univariable and multivariable analyses. We estimate that centralization to an HVC would result in 333 fewer nonroutine discharges (50\% reduction), 12,000 fewer hospital days (44\% reduction), and 165 fewer readmissions within 90 days (43\%). Treatment at HVCs would be cost-effective, saving \$15.3 million (11\% reduction) over the 5-year study time frame.

\section{Discussion}

This is the first study to describe nationwide readmission rates, causes, risk factors, complications, and outcomes associated with unplanned readmissions following EC-IC bypass surgery in the US. Among 2500 patients who underwent EC-IC bypass surgery, we report 30dRA and $90 \mathrm{dRA}$ rates of $7.5 \%$ and $14.0 \%$, respectively. These rates may serve as benchmarks for measuring the effects of future interventions to reduce readmissions. Since the 30dRA metric has been increasingly scrutinized in the recent literature for underestimating the true burden of postoperative readmissions, ${ }^{5-7}$ we also included the 90dRA metric (includes the 30-day window). Readmission rates for patients who had undergone an EC-IC bypass were similar to those for other neurosurgical procedures when comparing 30dRA and 90dRA..$^{5-8}$ Specific procedures include elective endovascular embolization (30dRA: 7.1\%, 90dRA: $13.5 \%$ ), transsphenoidal pituitary surgery (30dRA: 10.4\%), elective aneurysm clipping (30dRA: $9.1 \%, 90 \mathrm{dRA}: 14.9 \%),{ }^{5}$ craniotomy for malignant brain tumor (30dRA: 13.2\%, 90dRA: $25.7 \%),{ }^{6}$ acoustic neuroma surgery (30dRA: $7.7 \%, 90 \mathrm{dRA}: 9.1 \%),{ }^{7}$ and epilepsy surgery (30dRA: $11.5 \%, 90 \mathrm{dRA}: 16.8 \%){ }^{8}$

\section{Hospital EC-IC Bypass Case Volume and Quality Outcomes}

The EC-IC bypass is a technically challenging procedure with a steep learning curve. Furthermore, the perioperative care of these patients is complex and requires a multidisciplinary effort. ${ }^{18}$ HVCs are equipped with experienced personnel and appropriate resources for handling such complex surgical cases. In our population-level na-

TABLE 4. Hospital stratification by case volume of EC-IC bypass surgeries

\begin{tabular}{cccc}
\hline $\begin{array}{c}\text { Hospital EC-IC Bypass } \\
\text { Vol Percentile }\end{array}$ & $\begin{array}{c}\text { Min No. of Cases/Yr by Hospital } \\
\text { in Percentile Bracket }\end{array}$ & $\begin{array}{c}\text { Average No. of Cases/Yr by } \\
\text { Hospital Percentile Bracket }\end{array}$ & $\begin{array}{c}\text { Proportion of Total Patients Treated by } \\
\text { Hospitals in Each Percentile Bracket }\end{array}$ \\
\hline 99th or above & 25 & 29 & $13 \%$ \\
\hline 95th or above & 8 & 16 & $24 \%$ \\
\hline 90th or above & 6 & 12 & $33 \%$ \\
\hline 75th or above & 3 & 8 & $40 \%$ \\
\hline 50th or above & 2 & 6 & $52 \%$ \\
\hline
\end{tabular}


TABLE 5. Hospital volume of EC-IC bypass surgeries and perioperative stroke, postdischarge ischemic stroke, and readmission rate

\begin{tabular}{ccccc}
\hline $\begin{array}{c}\text { Hospital EC-IC Bypass } \\
\text { Vol Percentile }\end{array}$ & $\begin{array}{c}\text { Periop } \\
\text { Stroke (\%) }\end{array}$ & $\begin{array}{c}\text { Ischemic Stroke w/in 90 } \\
\text { Days of Discharge (\%) }\end{array}$ & 30dRA \% & 90dRA \% \\
\hline 99th or above & 6.5 & 0.7 & 2.9 & 5.6 \\
\hline 95th or above & 5.4 & 0.9 & 5.2 & 10.2 \\
\hline 90th or above & 6.0 & 1.8 & 5.5 & 10.6 \\
\hline 75th or above & 6.5 & 2.0 & 6.9 & 12.7 \\
\hline 50th or above & 6.5 & 2.2 & 7.7 & 14.4 \\
\hline
\end{tabular}

tionwide report, only $17 \%$ of patients were treated at HVCs (10 or more cases/year) and only approximately half of the patients were treated at hospitals performing two or more bypasses annually. Most clinical series have reported outcomes data from only highly experienced centers, which may underestimate the true rate of complications as well as morbidity and mortality.

Our results suggest that the centralization of EC-IC bypass surgery to HVCs would improve a wide range of patient outcomes, including readmission rates, while modestly decreasing the overall cost to the healthcare system. Treatment at HVCs was associated with significantly lower rates of perioperative complications, nonroutine discharge dispositions, prolonged LOSs, and readmission. The strength of this inverse relationship between bypass volume and readmission rate increased exponentially for hospitals in the 90th, 95th, and 99th percentiles for case volume, respectively.

Our findings are substantiated by prior analyses of the NIS that demonstrated a robust volume-outcome relationship., ${ }^{9,18,19}$ The NIS, unlike the NRD, does not track patients beyond the index hospitalization. Thus, the NIS cannot capture readmissions or postdischarge complications pertinent to bypass, such as ischemic stroke. Amin-Hanjani et al. previously studied a cohort of 588 admissions for ECIC bypass surgery from 1992 to 2001. ${ }^{9}$ The authors found that the majority of operations had been performed at lowvolume centers ( $<3$ cases/year). For hospitals performing at least one EC-IC bypass procedure between 1992 and 2001, the average annual bypass case volume was only 0.4 . The authors reported that HVCs were associated with a significantly lower likelihood of a nonroutine discharge disposi- tion (OR 0.45 for 10-fold increase in caseload). AkbarianTefaghi et al. analyzed a cohort of 10,679 admissions for EC-IC bypass between 2001 and 2014 in the NIS. ${ }^{18}$ They found that bypass at HVCs (4 or more cases per year) was associated with decreased mortality, fewer hospital days, and a lower rate of perioperative complications. ${ }^{18}$ Davies and Lawton identified a strong volume-outcome relationship in 8227 patients treated for cerebrovascular malformations in the NIS. ${ }^{19}$ Treatment at HVCs was similarly associated with significantly improved index hospitalization outcomes including shorter LOSs, lower complication rates, higher rates of discharge to home, and reduced hospital costs.

\section{Readmission and Postoperative Complications}

The longitudinal design of the NRD allowed for population-level estimates of postoperative complications beyond the index hospitalization for bypass surgery. The most common reasons for readmission in our analysis were similar for 30dRA and 90dRA including perioperative stroke, wound complications, and graft failure.

Our reported perioperative ischemic stroke rate at the index visit was $5.9 \%$, which is similar to the rate reported $(6.9 \%)$ in a prior NIS analysis by Winkler et al. ${ }^{10}$ Intraoperative vessel manipulation, temporary clipping, vessel injury, or undergoing anesthesia with fluctuations in blood pressure can contribute to this complication. ${ }^{20}$

Saber et al. investigated trends in the utilization and safety of EC-IC bypass surgery in 346 patients with MMD or ICAD. ${ }^{21}$ They reported a 30-day rate of combined stroke, hemorrhage, or death of 5.49\% (range 2.8\%$16.6 \%$ ) by year, which is similar to our rate of $6.1 \%$. Another study showed a similar 30-day stroke rate of $4.2 \% .^{22}$

TABLE 6. Univariable analysis: HVCs and outcomes for EC-IC bypass surgery

\begin{tabular}{lccc}
\hline \multicolumn{1}{c}{ Variable } & Non-HVC & HVC $(\geq 10$ cases/yr) & p Value \\
\hline Total no. of patients (\%) & $2066(82.6)$ & $434(17.4)$ & \\
\hline Periop stroke (\%) & 6.2 & 4.7 & 0.46 \\
\hline Ischemic stroke w/in 90 days of discharge (\%) & 2.9 & 0.8 & 0.03 \\
\hline Nonroutine discharge (\%) & 26.7 & 13.4 & 0.004 \\
\hline LOS in days & 10.9 & 6.1 & $<0.001$ \\
\hline Hospital costs in US\$ & 47,457 & 44,385 & 0.37 \\
\hline 30dRA (\%) & 8.2 & 3.9 & 0.03 \\
\hline 90dRA (\%) & 15.2 & 8.6 & 0.01 \\
\hline
\end{tabular}

Boldface type indicates statistical significance. 
TABLE 7. Multivariable analysis: HVCs and outcomes for EC-IC bypass surgery

\begin{tabular}{lccc}
\hline \multicolumn{1}{c}{ Binary Outcome Variable* } & OR for HVCs & $95 \% \mathrm{Cl}$ & $\mathrm{p} \mathrm{Value}$ \\
\hline Nonroutine discharge & 0.54 & $0.34-0.86$ & $<0.001$ \\
\hline Prolonged LOS $(>75$ th percentile for days) & 0.57 & $0.33-0.98$ & $\mathbf{0 . 0 4 2}$ \\
\hline Increased index visit costs $(>75$ th percentile for US\$) & 1.13 & $0.70-1.86$ & 0.61 \\
\hline 30dRA & 0.43 & $0.20-0.91$ & $\mathbf{0 . 0 2 7}$ \\
\hline 90dRA & 0.54 & $0.33-0.89$ & $\mathbf{0 . 0 1 6}$ \\
\hline
\end{tabular}

Boldface type indicates statistical significance.

* Each row represents separate binary logistic regression models. The models were each adjusted for potential con-

founders including patient age, comorbidity score, insurance type, and primary diagnosis.

With a focus on ICAD, our reported periprocedural stroke rate of $6.1 \%$ for patients after failed medical treatment was comparable to that in the control group in the Carotid Occlusion Surgery Study (COSS). ${ }^{3}$ The COSS was not able to show that EC-IC bypass surgery upfront decreased the stroke risk for ICAD patients, with a stroke risk of $14 \%{ }^{3}$ However, our results support the safety of EC-IC bypass surgery if medical treatment fails in ICAD patients.

The rate of readmission-associated graft failure in our study was $1.5 \%$. This was similar to the rate of graft failure in previously published studies such as the COSS with $2 \%$ graft failure at 30 days and $4 \%$ at the last follow-up (approximately 2 years). ${ }^{3}$ In a series of 430 bypass procedures, 12 bypass occlusions (2.8\%) occurred within 1 week of revascularization, ${ }^{23}$ and in a series of 47 patients who had undergone EC-IC bypass, diabetes and hyperlipidemia were found to be risk factors for poor development of the bypass graft. ${ }^{24}$

Wound complications are rare in craniotomies because of the redundancy of the rich vascular network to the scalp. However, the EC-IC bypass procedure requires the harvest and diversion of a major scalp vessel, commonly the superior temporal artery (STA), to the brain surface. This poses a potential risk for wound-related complications. In our study, we found a wound complication rate of $3.6 \%$ (1.2\% during index visit, $2.5 \%$ during readmission), which is lower than previously reported. Takanari et al.

TABLE 8. Estimated impact of centralization of care for EC-IC bypass surgery to HVCs

\begin{tabular}{|c|c|c|c|c|}
\hline Outcome Variable & Non-HVC & $\mathrm{HVC}^{*}$ & Difference & Reduction \\
\hline $\begin{array}{l}\text { Nonroutine } \\
\text { discharge, no. }\end{array}$ & 668 & 335 & 333 & $49.9 \%$ \\
\hline Index LOS in days & 27,250 & 15,250 & 12,000 & $44.0 \%$ \\
\hline 30dRA, no. & 205 & 98 & 107 & $52.2 \%$ \\
\hline 90dRA, no. & 380 & 215 & 165 & $43.4 \%$ \\
\hline Index visit costs in $\$ M$ & 118.6 & 111.0 & 7.6 & $6.4 \%$ \\
\hline $\begin{array}{l}\text { Readmission visit } \\
\text { costs in } \$ M\end{array}$ & 17.7 & 10.0 & 7.7 & $43.5 \%$ \\
\hline Total costs in \$M & 136.3 & 121 & 15.3 & $11.2 \%$ \\
\hline
\end{tabular}

detected 21 wound complications in 71 EC-IC bypass patients (29.6\%) including 7 severe cases. ${ }^{25}$ As expected, risk factors included preexisting diabetes and the harvesting of two versus one STA branch. Our study was limited to the capture of complication diagnoses in the ambulatory setting. Furthermore, while our ICD-9 coding definition was broad (e.g., nonhealing, disruption of wound, infection, seroma, dehiscence), many minor wound complications are likely not entered as coded diagnoses in the medical record. Thus, our rates are likely underestimates, representing only severe wound complications.

\section{Risk Factors for Readmission}

The identification of risk factors for readmission may guide future quality improvement initiatives aimed at reducing readmission rates. Furthermore, preoperative risk stratification is crucial for optimal patient selection. Patients who had undergone bypass for ICAD (vs MMD) had higher rates of readmission along with more complications of postoperative stroke, graft failure, and infection. We suspect that this disparity is likely attributable to an increased burden of vascular comorbidities in these patients including tobacco smoking, dyslipidemia, diabetes, hypertension, and peripheral vascular disease. After adjusting for age and preexisting comorbidity in the multivariable analysis, bypass indication for ICAD (vs MMD) was no longer associated with readmission.

We also observed higher readmission rates in patients with a Medicaid payer status, preexisting comorbidities (diabetes, hypertension, peripheral vascular disease, chronic kidney disease), and an adverse discharge disposition. The association between Medicaid payer and readmission is well described in other patient populations and commonly attributed to poor social support and lack of home resources for postoperative care. ${ }^{26}$ The interaction between socioeconomic status and healthcare outcomes is, however, multifactorial and outside the scope of this study. Discharge disposition, a marker of functional status, may be influenced by preexisting health status, disease severity, in-hospital interventions, and perioperative complications. It is intuitive that patients discharged to short-term or longterm care facilities would be more likely to be readmitted than the patients discharged to home. Vascular comorbidities, such as hypertension, diabetes, and hyperlipidemia, may drive readmission rates through increased rates of ischemic stroke, poor wound healing, and graft failure. Close outpatient follow-up for the management of medi- 
cal comorbidities, such as diabetes and hypertension, may reduce readmissions and associated complications, such as wound infections and stroke, following the bypass procedure. A recent systematic review and meta-analysis of postoperative stroke in MMD patients who had undergone bypass identified several risk factors: diabetes, preoperative ischemic events, and indirect bypass techniques. $.^{27} \mathrm{Hy}-$ pertension, age at onset, and sex were not associated with stroke. In our study, readmissions (and associated complications) were higher in patients with diabetes and hypertension, but the NRD cannot distinguish between indirect and direct bypass procedures.

\section{Study Limitations}

The present report is subject to the limitations of large administrative database studies. The NRD identifies diagnoses and procedures using ICD-9/-10 codes and is subject to variations in coding practices and accuracy. Furthermore, ICD codes have limited granularity for identifying specifics regarding preadmission functional status, clinical examinations, radiological data, pharmacotherapy, and operative details. Our results are only generalizable for bypass procedures in general because our data do not differentiate between different bypass procedures such as direct versus indirect techniques or EC-IC versus IC-IC anastomosis. The NRD does not include timing for various diagnoses or procedures. Thus, our results are reflective of "independent associations" and do not imply causality. We are unable to determine if a complication or readmission is directly related to the surgical procedure. The NRD does not track readmissions across years, which limits duration of follow-up. Despite these limitations, a longitudinal database of this magnitude is optimal for studying readmissions and associated complications for relatively uncommon diagnoses and procedures, such as MMD and EC-IC bypass surgery. The database has a wealth of demographic diversity in terms of socioeconomic status, geographic region, and hospital type. In the clinical literature, randomized controlled trials are heralded as the highest level of medical evidence. However, especially when studying surgical outcomes, the design of a randomized controlled trial is sometimes financially impractical, unethical, or unfeasible. Nationwide database studies are important for hypothesis generation prior to the design of targeted prospective studies and the creation of multicenter registries.

\section{Conclusions}

Here, we report the first nationwide study of readmissions following EC-IC bypass surgery for MMD, ICAD, or unruptured aneurysm. Readmission rates were comparable to those for other common cranial procedures including craniotomy for brain tumors. Readmission visits captured several postoperative complications including stroke, graft failure, and wound complications. Readmissions are primarily driven by patient age, socioeconomic status, preexisting comorbidities, and treatment at low-volume centers. Only $17 \%$ of patients were treated at HVCs (10 or more EC-IC bypass cases annually). Those treated at HVCs had significantly lower rates of a nonroutine discharge dispo- sition, prolonged hospital stay, readmission, and ischemic stroke within 90 days. The centralization of care may significantly reduce the operative morbidity associated with the technically challenging EC-IC bypass procedure.

\section{References}

1. Burkhardt J-K, Lawton MT. Practice trends in intracranial bypass surgery in a 21-year experience. World Neurosurg. 2019;125:e717-e722.

2. Lawton MT. Seven Bypasses: Tenets and Techniques for Revascularization. Thieme; 2018.

3. Powers WJ, Clarke WR, Grubb RL Jr, et al. Extracranialintracranial bypass surgery for stroke prevention in hemodynamic cerebral ischemia: the Carotid Occlusion Surgery Study randomized trial. JAMA. 2011;306(18):19831992.

4. EC/IC Bypass Study Group. Failure of extracranial-intracranial arterial bypass to reduce the risk of ischemic stroke. Results of an international randomized trial. $N$ Engl J Med. 1985;313(19):1191-1200.

5. Hoffman H, Protas M, Chin LS. A nationwide analysis of 30-day and 90-day readmissions after elective cerebral aneurysm clipping in the United States: causes, predictors, and trends. World Neurosurg. 2019;128:e873-e883.

6. Donoho DA, Wen T, Babadjouni RM, et al. Predictors of 30and 90-day readmission following craniotomy for malignant brain tumors: analysis of nationwide data. J Neurooncol. 2018;136(1):87-94.

7. Babadjouni R, Wen T, Donoho DA, et al. Increased hospital surgical volume reduces rate of 30- and 90-day readmission after acoustic neuroma surgery. Neurosurgery. 2019;84(3): 726-732.

8. Rumalla K, Smith KA, Arnold PM, Schwartz TH. Readmission following surgical resection for intractable epilepsy: nationwide rates, causes, predictors, and outcomes. Oper Neurosurg (Hagerstown). 2019;16(3):374-382.

9. Amin-Hanjani S, Butler WE, Ogilvy CS, et al. Extracranialintracranial bypass in the treatment of occlusive cerebrovascular disease and intracranial aneurysms in the United States between 1992 and 2001: a population-based study. J Neurosurg. 2005;103(5):794-804.

10. Winkler EA, Yue JK, Deng H, et al. National trends in cerebral bypass surgery in the United States, 2002-2014. Neurosurg Focus. 2019;46(2):E4.

11. Sun H, Kalakoti P, Sharma K, et al. Proposing a validated clinical app predicting hospitalization cost for extracranialintracranial bypass surgery. PLoS One. 2017;12(10):e0186758.

12. Hoffman H, Protas M, Chin LS. Causes, predictors, and trends of unplanned readmissions after elective endovascular embolization of cerebral aneurysms. J Stroke Cerebrovasc Dis. 2019;28(11):104396.

13. Buchanan IA, Donoho DA, Patel A, et al. Predictors of surgical site infection after nonemergent craniotomy: a Nationwide Readmission Database analysis. World Neurosurg. 2018; 120:e440-e452.

14. Koo AB, Elsamadicy AA, David WB, et al. Thirty- and 90-day readmissions after treatment of traumatic subdural hematoma: national trend analysis. World Neurosurg. 2020;139:e212-e219.

15. Adhikari S, Hossein MZ, Das A, et al. Etiology and outcome of acute intestinal obstruction: a review of 367 patients in Eastern India. Saudi J Gastroenterol. 2010;16(4):285-287.

16. Janjua N, Nasar A, Lynch JK, Qureshi AI. Thrombolysis for ischemic stroke in children: data from the nationwide inpatient sample. Stroke. 2007;38(6):1850-1854.

17. Tang AM, Bakhsheshian J, Ding L, et al. Nonindex readmission after ruptured brain aneurysm treatment is associated with higher morbidity and repeat readmission. World Neurosurg. 2019;130:e753-e759. 
18. Akbarian-Tefaghi H, Kalakoti P, Sun H, et al. Impact of hospital caseload and elective admission on outcomes after extracranial-intracranial bypass surgery. World Neurosurg. 2017;108:716-728.

19. Davies JM, Lawton MT. Improved outcomes for patients with cerebrovascular malformations at high-volume centers: the impact of surgeon and hospital volume in the United States, 2000-2009. J Neurosurg. 2017;127(1):69-80.

20. Kazumata K, Ito M, Tokairin K, et al. The frequency of postoperative stroke in moyamoya disease following combined revascularization: a single-university series and systematic review. J Neurosurg. 2014;121(2):432-440.

21. Saber H, Rajah G, Palla M, Sheth SA. Utilization and safety of extracranial-intracranial bypass surgery in symptomatic steno-occlusive disorders. Brain Circ. 2019;5(1):32-35.

22. von Weitzel-Mudersbach P, Andersen G, Rosenbaum S. Low morbidity after extracranial-intracranial bypass operation. The Danish Extracranial-Intracranial Bypass Study: a nationwide survey. Cerebrovasc Dis. 2018;45(5-6):252-257.

23. Yoon S, Burkhardt J-K, Lawton MT. Long-term patency in cerebral revascularization surgery: an analysis of a consecutive series of 430 bypasses. J Neurosurg. 2018;131(1):80-87.

24. Nomura S, Yamaguchi K, Ishikawa T, et al. Clinical factors influencing the development of extracranial-intracranial bypass graft for steno-occlusive cerebrovascular disease. $\mathrm{Neu}$ rosurg Focus. 2019;46(2):E5.

25. Takanari K, Araki Y, Okamoto, et al. Operative wound-related complications after cranial revascularization surgeries. $J$ Neurosurg. 2015;123(5):1145-1150.
26. Basu J, Friedman B, Burstin H. Preventable hospitalization and Medicaid managed care: does race matter? J Health Care Poor Underserved. 2006;17(1):101-115.

27. Wei W, Chen X, Yu J, Li X-Q. Risk factors for postoperative stroke in adults patients with moyamoya disease: a systematic review with meta-analysis. BMC Neurol. 2019;19(1):98.

\section{Disclosures}

The authors report no conflict of interest concerning the materials or methods used in this study or the findings specified in this paper.

\section{Author Contributions}

Conception and design: Burkhardt, Rumalla. Acquisition of data: Rumalla. Analysis and interpretation of data: all authors. Drafting the article: Rumalla. Critically revising the article: all authors. Reviewed submitted version of manuscript: all authors. Statistical analysis: Rumalla, Gaddis. Administrative/technical/material support: Burkhardt. Study supervision: Burkhardt.

\section{Correspondence}

Jan-Karl Burkhardt: Hospital of the University of Pennsylvania, Philadelphia,PA. jan.burkhardt@pennmedicine.upenn.edu. 